\title{
A revised method to calculate time-varying mesh stiffness of helical gear
}

\author{
Xiao $\mathrm{Wu}^{1}$, Yang Luo ${ }^{1, *}$, Qinmin $\mathrm{Li}^{1}$, and Juanjuan $\mathrm{Shi}^{2}$ \\ ${ }^{1}$ College of Mechanical Engineering, Chongqing University, Chongqing 400030, China \\ ${ }^{2}$ School of Rail Transportation, Soochow University, Suzhou 215131, China
}

\begin{abstract}
Time-varying mesh stiffness (TVMS) of gear plays vital role in analysing dynamic characteristic of gear transmission. So accurately evaluating the TVMS is important and essential. In this paper, a revised method to calculate the TVMS of helical gear is proposed. Based on slice method, the helical gear is sliced into pieces along the tooth width direction. The proposed method corrects the fillet foundation stiffness within multi-tooth in contact and considers the non-linearity and loaddependence of the Hertzian contact stiffness. The effect of the axial mesh force is considered. Finally, an equivalent helical gear model is established in ANSYS to study the mesh stiffness. The results show the proposed method has high effectiveness compared with FEM (finite element method).
\end{abstract}

Keywords: Time-varying mesh stiffness, Helical gear, FEM, Slice method.

\section{Introduction}

Gear transmission is mostly applied in motion and power transmission [1-3]. Timevarying mesh stiffness (TVMS) of gear plays vital role in gear transmission and dynamic analysis. Therefore, accurate evaluation the TVMS of gears is essential in analysing the dynamic behaviours of a transmission [4].

Much research has been carried out to investigated TVMS of both spur and helical gears. Two most commonly methods are analytical method (AM) and finite element method (FEM). FEM has been widely utilized to evaluate the TVMS for its high accuracy. Wang and Howard [5] used finite element method to analysis the torsional stiffness of involute spur gear. Jyothirmai [6] studied the several helical gear tooth systems including single, herringbone and crossed helical gear by finite element method and compared with theoretical analysis. Ma et al. [7] utilized the FEM to evaluate the TVMS of spur gear under tooth spalling defects. Liang et al. [8] proposed three new models to evaluate the mesh stiffness of involute spur gear.

${ }^{*}$ Corresponding author: yluo688@equ.edu.cn 
AM has been considered to be the most useful method to calculate TVMS. Yang and Lin [9] proposed the potential energy and modelled the gear tooth as a cantilever of variable cross-section, with considering of Hertzian contact stiffness, bending stiffness and axial compressive stiffness to calculate the TVMS of gear pairs. Tian et al. [10] considering shear stiffness based the above theory. This work has been improved by Zhou et al. [11] and Wan et al. [12] through considering the fillet foundation stiffness. Luo et al. [13] proposed a new gear mesh kinematic model to accurately evaluate the contact position of tooth engagement and calculated the TVMS of spur gear under the perfect mounting and constant center distance deviation. Xie et al. [14] revised the analytical calculation method of gear body-induced tooth deflections with considering structure coupling effect. The result was validated by FEM and other formular. Later, Xie et al. [15] further established a mesh stiffness model and a load sharing model and corrected the fillet foundation stiffness.

Based on slice method, Wan et al. [16] proposed accumulated integral potential energy method to compute the TVMS of helical gear by dividing gear into many slices. Wang et al. [17] considered the influence of axial mesh force. Tang et al. [18] and Wei et al. [19] introduced single coupled model and two coupled model which correct the error caused by the each slice gear, respectively. Yu et al. [19] further analysed the relationship of each two sliced gear and brought weight factor to revise the result.

In view of the above, this paper proposes a revised method to evaluate the TVMS of helical gear. Based on slice method, the helical gear is sliced into pieces along the tooth width direction. The mesh stiffness of each slice gear can be evaluated. The proposed method corrects the fillet foundation stiffness within the multi-tooth contact and the Hertzian contact stiffness due to its nonlinear and load-dependent. According to the force distribution of the gear in contact, the effect of the axial mesh stiffness is taken into consideration. Finally, the effectiveness of the results from proposed method is validated by finite element method.

\section{Evaluation of the time-varying mesh stiffness of helical gear}

\subsection{The TVMS of single tooth pair}

Due to the existence of non-zero helical angle, the structure of helical gear is different from spur gear. The computation method of spur gear to evaluate the mesh stiffness cannot be directly applied to that of helical gear. For helical gear, the slice method is generally utilized to overcome this shortcoming, which the helical gear tooth is sliced into pieces along the tooth width direction, and each piece is approximately as a very thin spur gear (see figure 1). Thus, the helical gear can be seen as a series of spur gear.

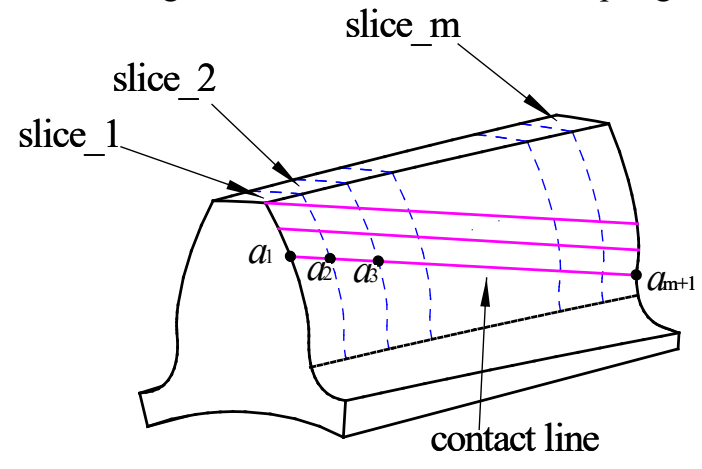

Fig. 1. The slice model of helical gear. 


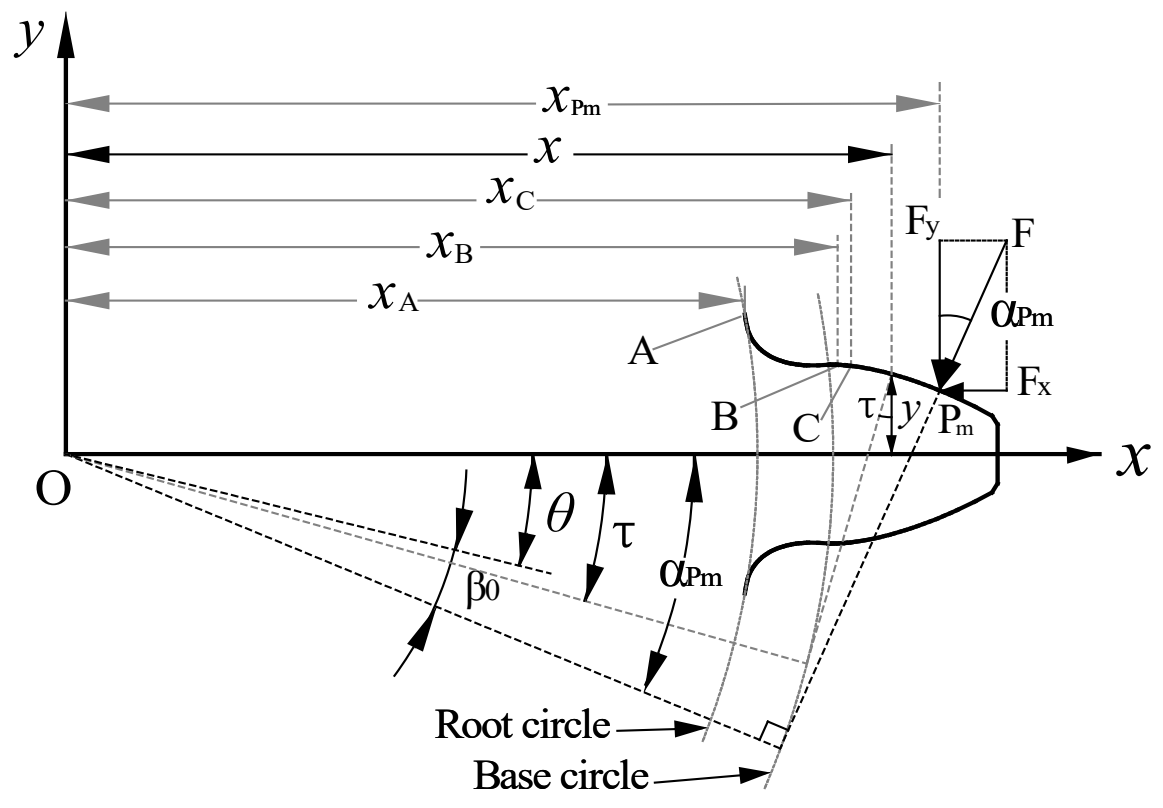

Fig. 2. The geometric model for evaluating the TVMS of spur gears[13].

Based on potential energies, gear tooth is modelled as a cantilever beam with variable cross-section (see figure 2). And the total energy of the slice tooth is the sum of bending energy, axial compressive energy, shear energy, fillet foundation energy and Hertzian energy, which can be expressed as:

$$
\left\{\begin{array}{l}
d U_{a}=\frac{F^{2}}{2 d k_{a}}=\int_{x_{A}}^{x_{B}} \frac{F_{x}^{2}}{2 E d A_{x 1}} d x_{1}+\int_{x_{B}}^{x_{p m p}} \frac{F_{x}^{2}}{2 E d A_{x 2}} d x_{2}, \\
d U_{b}=\frac{F^{2}}{2 d k_{b}}=\int_{x_{A}}^{x_{B}} \frac{M_{1}^{2}}{2 E d I_{y 1}} d x_{1}+\int_{x_{B}}^{x_{p m}} \frac{M_{2}^{2}}{2 E d I_{y 2}} d x_{2}, \\
d U_{s}=\frac{F^{2}}{2 d k_{s}}=\int_{x_{A}}^{x_{B}} \frac{1.2 F_{y}^{2}}{2 G d A_{x 1}} d x_{1}+\int_{x_{B}}^{x_{p m}} \frac{1.2 F_{y}^{2}}{2 G d A_{x 2}} d x_{2}, \\
d U_{f}=\frac{F^{2}}{2 d k_{f}}, \\
d U_{h}=\frac{F^{2}}{2 d k_{h}},
\end{array}\right.
$$

Here $E, G$ are Young's modulus and shear modulus, respectively. $F$ is the teeth contact force, and the force distribution of the helical gear in meshing is shown in figure 3. $F_{t}=$ $F \cos \beta, F_{x}=F_{t} \sin \alpha_{p m}, F_{y}=F_{t} \cos \left(\alpha_{p m}\right)$, and $\beta$ is helix angel. The axial compressive stiffness, bending stiffness, shear stiffness can be expressed as: 


$$
\left\{\begin{array}{l}
\frac{1}{d k_{a}}=\int_{\frac{\pi}{2}}^{\alpha_{0}} \frac{\cos ^{2} \beta \sin ^{2} \alpha_{p m}}{E d A_{x_{1}}} \cdot \frac{d x_{1}}{d \gamma} d \gamma+\int_{\alpha_{B}}^{x_{p m}} \frac{\cos ^{2} \beta \sin ^{2} \alpha_{p m}}{E d A_{x_{2}}} \cdot \frac{d x_{2}}{d \tau} d \tau, \\
\frac{1}{d k_{b}}=\int_{\frac{\pi}{2}}^{\alpha_{0}} \frac{\left(\cos \beta \cdot \cos \alpha_{p m} \cdot\left(x_{p m}-x_{1}\right)-\cos \beta \cdot \sin \alpha_{p m} y_{p m}\right)^{2}}{E d I_{y_{1}}} \cdot \frac{d x_{2}}{d \gamma} d \gamma, \\
+\int_{\alpha_{B}}^{x_{p m}} \frac{\left(\cos \beta \cdot \cos \alpha_{p m} \cdot\left(x_{p m}-x_{2}\right)-\cos \beta \cdot \sin \alpha_{p m} y_{p m}\right)^{2}}{E d I_{y_{2}}} \cdot \frac{d x_{2}}{d \tau} d \tau, \\
\frac{1}{d k_{s}}=\int_{\frac{\pi}{2}}^{\frac{\alpha_{0}}{2}} \frac{1.2 \cos ^{2} \beta \cos ^{2} \alpha_{p m}}{G d A_{x_{1}}} \cdot \frac{d x_{1}}{d \gamma} d \gamma+\int_{\alpha_{B}}^{x_{p m}} \frac{1.2 \cos ^{2} \beta \cos ^{2} \alpha_{p m}}{G d A_{x_{2}}} \cdot \frac{d x_{2}}{d \tau} d \tau,
\end{array}\right.
$$

where $d A_{x 1}=2 y_{t r} d W, d A_{x 2}=2 y_{p} d W, d I_{x 1}=2 / 3 y_{t r}^{3} d W, d A I_{x 2}=2 / 3 y_{p}^{3} d W$ are the cross-sectional areas and area moments of inertia of a slice tooth, and $d W$ is the width of each slice tooth. $M_{1}, M_{2}, x_{1}, x_{2}, y_{t r}, y_{p}, \frac{d x_{1}}{d \gamma}, \frac{d x_{2}}{d \tau}$ are listed in Reference.[13].

For the calculation of fillet foundation stiffness and Hertzian contact stiffness, the traditional analytical (TA) adopted the analytical formula which can be expressed as[16]:

$$
\begin{gathered}
\frac{1}{d k_{f}}=\frac{\cos ^{2} \alpha_{\mathrm{m}}}{E d W}\left\{L^{*}\left(\frac{\mu_{f}}{S_{f}}\right)^{2}+M^{*}\left(\frac{\mu_{f}}{S_{f}}\right)+P^{*}\left(1+Q^{*} \tan ^{2} \alpha_{m}\right)\right\} \\
d k_{h}=\frac{\pi E d W}{4\left(1-v^{2}\right)}
\end{gathered}
$$

here $u_{f}, S_{f}, L^{*}, M^{*}, P^{*}, Q^{*}$ are the parameter to correct the fillet foundation stiffness which can be found in[20]. $v$ is the Poisson's ratio.

However, the Hertzian contact stiffness is load-dependent and nonlinear. Equation (3) is only suitable for single tooth pair in mesh. For multi-tooth pairs in mesh, equation (3) overestimates the mesh stiffness because multi-tooth pairs shared on gear body simultaneously. Langlois [21] proposed an analytical formula which can be expressed as:

$$
\begin{array}{r}
\frac{1}{d k_{f}}=\frac{\cos ^{2} \beta \cos ^{2} \alpha_{p m}}{E d W C_{f}^{*}}\left\{L^{*}\left(\frac{u_{f}}{S_{f}}\right)^{2}+M^{*}\left(\frac{u_{f}}{S_{f}}\right)+P^{*}\left(1+Q^{*} \tan ^{2} \alpha_{p m}\right)\right\} \\
d k_{h}=\frac{\pi E d W C_{h}^{*}}{4\left(1-v^{2}\right)}\left(\ln \left(\frac{2 \sqrt{h_{1 P m} h_{2 P m}}}{b_{P m}}\right)-\frac{v}{2(1-v)}\right)^{-1}, \text { with } b_{P m}=\sqrt{\frac{8 F\left(1-v^{2}\right) r_{1 P m} r_{2 P m}}{\pi d W E\left(r_{1 P m}+r_{2 P m}\right)}}
\end{array}
$$

$r_{1 P m}, r_{2 P m}, b_{P m}, h_{1 P m}, h_{2 P m}$ are parameters to compute the Hertzian contact stiffness which can be found in[21]. $C_{f}^{*}$ and $C_{h}^{*}$ are utilized to correct the filler foundation within the doubletooth pair meshing region and Hertzian contact stiffness. $C_{f}^{*}$ and $C_{h}^{*}$ can be obtained by finite element method. 
Based on potential energy, the total energy stored in a slice gear pair can be expressed as:

$$
d U=d U_{a}+d U_{b}+d U_{s}+d U_{f}+d U_{h}=\frac{F^{2}}{2 d k_{t n}}
$$

The mesh stiffness of a slice gear can be expressed as:

$$
d k_{t n}=1 /\left(\frac{1}{d k_{a 1}}+\frac{1}{d k_{b 1}}+\frac{1}{d k_{s 1}}+\frac{1}{d k_{f 1}}+\frac{1}{d k_{a 2}}+\frac{1}{d k_{b 2}}+\frac{1}{d k_{s 2}}+\frac{1}{d k_{f 2}}+\frac{1}{d k_{h}}\right)
$$

The total mesh stiffness of a slice gear pair can be expressed as:

$$
k_{t n}=\sum d k_{t n}
$$

However, equation (9) neglects the influence of axial mesh force. According to the force distribution of the helical gear [22], this paper adapts following equation to correct the error from axial mesh force:

$$
k_{t}=k_{t n} * \cos ^{2} \beta_{b}
$$

here $\beta_{b}$ is the helix angle of the base circle.

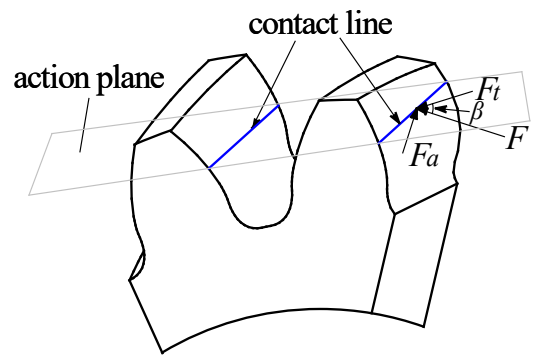

Fig. 3. The force distribution.

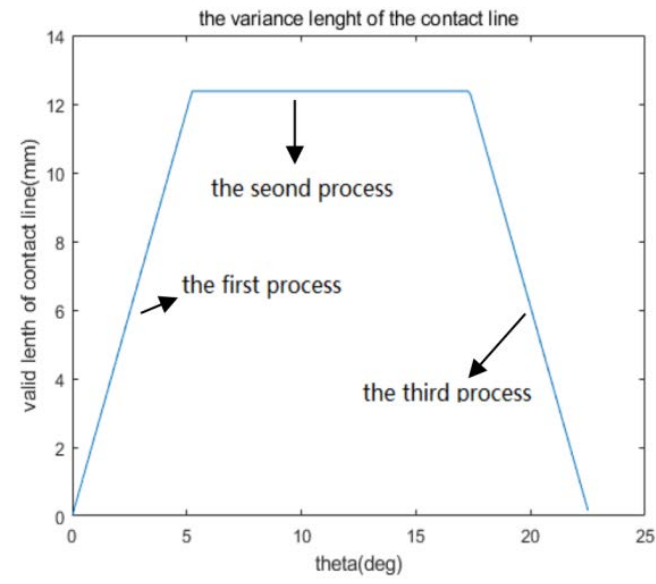

Fig. 4. The three processes of the contact line. 


\subsection{The total mesh stiffness of helical gear}

The mating process of a helical gear pair can be divided into three periods. At the beginning, the helical tooth pair starts to contact and the length of contact line increases gradually. Then, the length of contact line and stiffness keeps relatively unchanged. Finally, the length of contact line decreases until gear teeth loss of contact, as is shown in figure 4 . The total contact ratio of a helical gear pair $\varepsilon_{\gamma}$ is the sum of the transverse contact ratio $\varepsilon_{\alpha}$ and the face contact ratio $\varepsilon_{\beta}$, e.g., $\varepsilon_{\gamma}=\varepsilon_{\alpha}+\varepsilon_{\beta}$. The number of mating teeth pairs varies during the mating process, as is shown figure 5 , the red area represents maximum mating teeth pairs area while the cyan area is minimum mating teeth pairs area, $L_{1}=f \operatorname{loot}\left(\varepsilon_{\gamma}\right) \cdot P_{b t}$ in which $P_{b t}$ is the transverse base pitch. the total mesh stiffness of helical gear (see figure 6) can be expressed as:

$$
k=\sum_{j=1}^{p} k_{t}^{j}
$$

where $j, p$ represent the $i^{t h}$ pair and the responding mating teeth pairs, respectively.

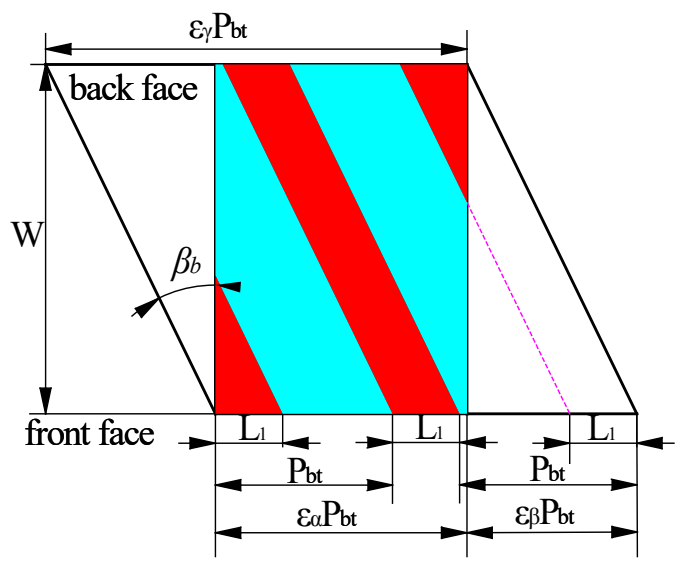

Fig. 5. The mating process of a helical gear pair.

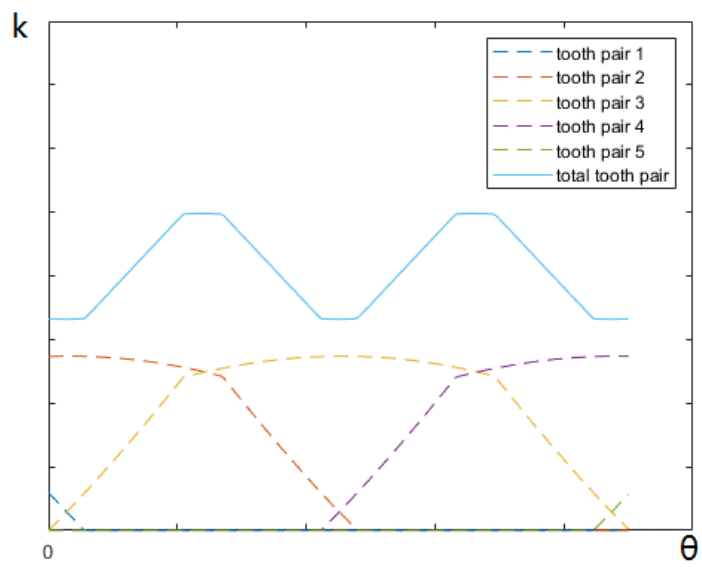

Fig. 6. Schematic diagram of the tooth pairs mesh stiffness. 


\section{Validation}

In order to validate the accuracy of the proposed method to evaluate the TVMS of helical gear, a finite element is established in ANSYS software (see figure 7). To improve the computational efficiency, only one tooth pair is involved. The parameters of the helical gear pair are listed in Table 1. For the driven gear, all the degrees of freedom are fully constrained. And the driving gear only has rotational degree of freedom along its axis. The torque $T$ is applied in the inner hubs of the driving gear in anticlockwise direction. The rotation deformation $\theta$ of the driving gear at the corresponding contact position can be obtained. And the torsional stiffness of single tooth pair can be expressed as:

$$
k_{t o}=\frac{T}{\theta}
$$

And the mesh stiffness of single tooth pair is then calculated by:

$$
k^{*}=\frac{k_{t o}}{R_{b 1}}
$$

here $R_{b 1}$ is the base radius of the driving gear.

\begin{tabular}{|c|c|c|c|}
\hline properties & symbol & driving gear & driven gear \\
\hline Normal module $(\mathrm{mm})$ & $m_{n}$ & 2 & \\
\hline Number of teeth & $N_{1}, N_{2}$ & 34 & 52 \\
\hline Helix angel $\left({ }^{\circ}\right)$ & $\beta$ & 15 & \\
\hline Face width $(\mathrm{mm})$ & $W$ & 12 & \\
\hline Normal pressure angel $\left({ }^{\circ}\right)$ & $\alpha_{n}$ & 20 & \\
\hline Young's modulus $(\mathrm{Pa})$ & $E$ & $2.0 * 10^{11}$ & \\
\hline Passion ratio & $v$ & 0.3 & \\
\hline Hub radius $(\mathrm{mm})$ & $r_{i}$ & 15 & \\
\hline Applied torque $(\boldsymbol{N} \cdot \boldsymbol{m})$ & $T$ & 100 & \\
\hline Contact ratio & $\varepsilon_{\gamma}$ & 2.1321 & \\
\hline
\end{tabular}

Table 1. The parameters of the helical gear pair.

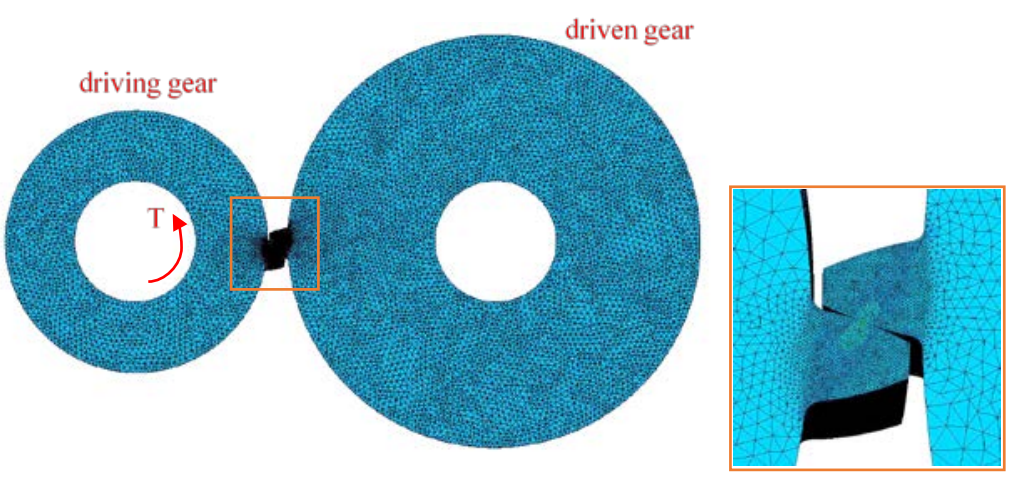

Fig. 7. The finite element model.

The single tooth pair TVMS of helical gear evaluated by proposed method (PM), traditional method (TM) and FEM is shown in figure 8(a). It shows that the single mesh 
stiffness calculated by proposed method is very close to FEM. However, the result from traditional method is much bigger than that from PM and FEM. The reason is that TM neglects that multi-tooth pair shared one body simultaneously in calculating fillet foundation stiffness and the Hertzian contact stiffness is load-dependent and nonlinear. The total TVMS of helical gear over one period calculated by those three methods in shown in figure 8(b). it shows the TVMS evaluated by PM is consistent with that by FEM.
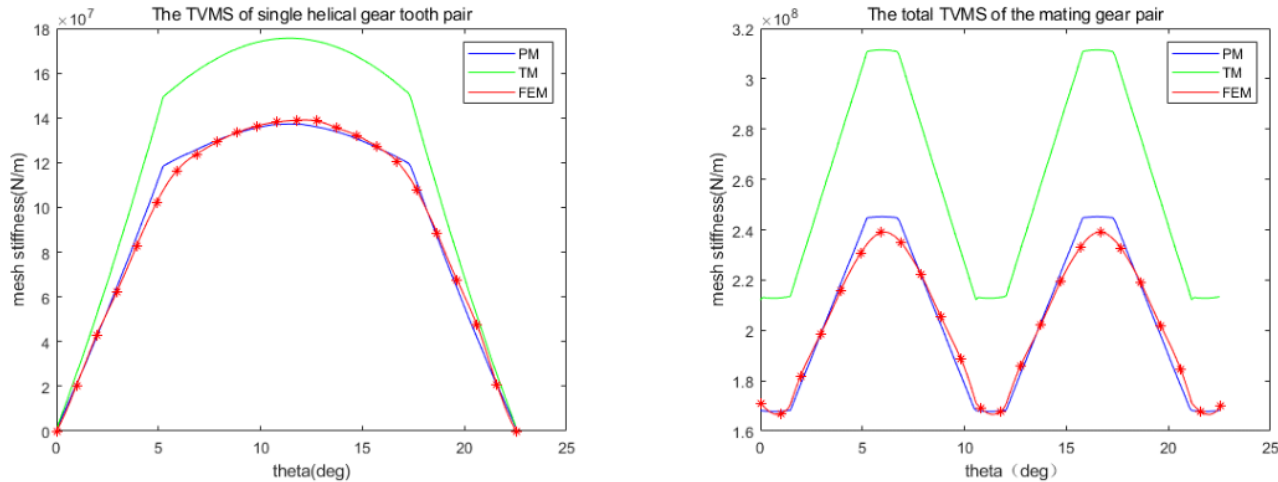

Fig. 8. (a) The TVMS of single helical gear tooth pair; (b) The total TVMS of the mating gear pair.

\section{Conclusion}

This paper proposes a revised method to evaluate the TVMS of helical gear. Based on slice method, this method corrects the fillet foundation stiffness in multi tooth pair meshing area and considers the nonlinear and load-dependent of Hertzian contact stiffness. Besides, the influence of the axial mesh force also takes into consideration. The accuracy of the proposed method is validated by the FEM. The results show the TVMS evaluated by PM is consistent with that by FEM both in single tooth pair mesh stiffness and multi tooth pair mesh stiffness.

This project was supported by the National Natural Science Foundation of China (No. 51905052, No. 51605319) and the Natural Science Foundation of Chongqing--Funds for Creative Research Groups (no. cstc2019jcyj-cxttX0003).

\section{References}

1. Mohammed O D, M Rantatalo and J-O Aidanpää 2013 Engineering Failure Analysis. 34 pp 235-251

2. Hedlund J and A Lehtovaara 2008 P I Mech Eng C-J Mec. 222 pp 1321-1327

3. Jiang H J, Y M Shao and C K Mechefske 2014 Eng Fail Anal. 39 pp 92-107

4. Liang X, M J Zuo and M Pandey 2014 Mechanism and Machine Theory. 76 pp 20-38

5. JWang and I Howard 2004 Proceedings of the Institution of Mechanical Engineers Part C-Journal of Mechanical Engineering Science. 218 pp 131-142

6. Jyothirmai S, R Ramesh, T Swarnalatha and D Renuka 2014 Procedia Materials Science. 6 pp 907-918

7. Ma H, Z Li, M Feng, R Feng and B Wen 2016 Engineering Failure Analysis. 66 pp $166-176$ 
8. Liang X, H Zhang, M J Zuo and Y Qin 2018 Mechanical Systems and Signal Processing. $101 \mathrm{pp}$ 424-434

9. Yang D C H and J Y Lin 1987 J Mech Transm-T Asme. 109 pp 189-196

10. Tian X, M J Zuo and K R Fyfe 2004 ASME 2004 International Mechanical Engineering Congress and Exposition. pp 785-793

11. Zhou X, Y Shao, Y Lei and M Zuo 2011 Journal of Vibration and Acoustics. 134

12. Wan Z G, H R Cao, Y Y Zi, W P He and Z J He 2014 Eng Fail Anal. 42 pp 157-177

13. Luo $Y, N$ Baddour and M Liang 2017 Engineering Failure Analysis. 75 pp 37-53

14. Xie C, L Hua, X Han, J Lan, X Wan and X Xiong 2018 International Journal of Mechanical Sciences. 148 pp 174-190

15. Xie C, L Hua, J Lan, X Han, X Wan and X Xiong 2018 Mechanical Systems and Signal Processing. 111 pp 331-347

16. Wan Z, H Cao, Y Zi, W He and Y Chen 2015 Mechanism and Machine Theory. 92 pp 447-463

17. Wang Q, B Zhao, Y Fu, X Kong and H Ma 2018 Mechanical Systems and Signal Processing. 106 pp 413-429

18. Tang X L, L Zou, W Yang, Y J Huang and H Wang 2018 Appl Math Model. 64 pp 524-540

19. Yu W and $\mathrm{C} \mathrm{K}$ Mechefske 2018 Iranian Journal of Science and Technology, Transactions of Mechanical Engineering. 43 pp 503-515

20. Sainsot, P., P Velex and O Duverger 2004 Journal of Mechanical Design. 126 pp 748752

21. Langlois P, B AI and O Harris 2016 Gear Technology.

22. Wang G 2018 Chongqing university 